\title{
E2F1 promotes tumor cell invasion and migration through regulating CD147 in prostate cancer
}

\author{
YU-XIANG LIANG ${ }^{1 *}$, JIAN-MING LU $^{1 *}$, RU-JUN MO $^{1 *}$, HUI-CHAN HE ${ }^{1}$, JIAN XIE $^{1}$, \\ FU-NENG JIANG ${ }^{1}$, ZHUO-YUAN LIN ${ }^{1}$, YAN-RU CHEN ${ }^{1}$, YONG-DING WU ${ }^{1}$, \\ HONG-WEI LUO ${ }^{1}$, ZHENG LUO $^{2}$ and WEI-DE ZHONG ${ }^{1,3,4}$
}

\begin{abstract}
${ }^{1}$ Department of Urology, Guangdong Key Laboratory of Clinical Molecular Medicine and Diagnostics, Guangzhou First People's Hospital, Guangzhou Medical University, Guangzhou, Guangdong 510180; ${ }^{2}$ School of Marine Sciences,

Sun Yat-sen University, Guangzhou, Guangdong 510275; ${ }^{3}$ Guangdong Provincial Institute of Nephrology, Southern Medical University, Guangzhou, Guangdong 510515; ${ }^{4}$ Urology Key Laboratory of Guangdong Province, The First Affiliated Hospital of Guangzhou Medical University, Guangzhou Medical University, Guangzhou, Guangdong 510230, P.R. China
\end{abstract}

Received October 6, 2015; Accepted January 11, 2016

DOI: $10.3892 /$ ijo.2016.3364

\begin{abstract}
Increased expression of E2F1 has been reported to be associated with tumor growth and cell survival of prostate cancer (PCa). However, its roles and mechanisms on PCa have not been fully elucidated. The present study found that E2F1 overexpression in PCa tissues was significantly associated with high Gleason score $(\mathrm{P}=0.01)$ and advanced pathological stage $(\mathrm{P}=0.02)$. In addition, $\mathrm{PCa}$ patients with high E2F1 expression more frequently had shorter biochemical recurrence-free survival $(\mathrm{P}=0.047)$ than those with low E2F1 expression. Then, we confirmed that the knock-down of E2F1 expression was able to inhibit cell cycle progression, invasion and migration of PCa cell lines in vitro, along with tumor xenograft growth and epithelialto-mesenchymal transition (EMT) in vivo. Moreover, we identified CD147 as a novel interaction partner for E2F1 through bio-informatic binding site prediction, combined with chromatin immunoprecipitation-PCR (ChIP-PCR) and western blot analysis. Taken together, our data delineate an as yet unrecognized function of E2F1 as enhancer of tumor invasion and migration of $\mathrm{PCa}$ via regulating the expression of CD147 in PCa. Importantly, E2F1 may function as a biomarker that can differentiate patients with biochemical recurrent and non-biochemical recurrent disease following radical prostatectomy, highlighting its potential as a therapeutic target.
\end{abstract}

Correspondence to: Dr Wei-De Zhong, Department of Urology, Guangzhou First People's Hospital, Guangzhou Medical University, Guangzhou, Guangdong 510180, P.R. China

E-mail: zhongwd2009@live.cn

*Contributed equally

Key words: prostate cancer, E2F1, tumor invasion, tumor migration, biochemical recurrence-free survival, CD147

\section{Introduction}

Prostate cancer $(\mathrm{PCa})$ represents the most common malignancy in males in the United States other than skin cancer and the second leading cause (after lung cancer) of cancer-related deaths (1). The majority of patients with PCa behave in an indolent manner, but a subset is highly aggressive (2). Locally defined disease is often successfully treated with surgery and/or radiotherapy; however, disease recurs in an estimated $15-30 \%$ of patients (3). Treatment decisions are currently based on clinical and histological features only. Although radical prostatectomy (RP) and radiation therapy are the most appropriate treatment for patients with localized PCa, the risk of biochemical disease recurrence in RP patients is $25 \%$ (4). Since PCa often progresses rapidly and most death is caused by metastases that are resistant to conventional therapies, the optimal therapy for patients with this cancer has yet to be defined. As the molecular etiology of PCa becomes better understood, it is clear that $\mathrm{PCa}$ progression is influenced by a multistep process, involving both genetic insults to epithelial cells and changes in epithelial-stromal interactions (5). Therefore, it is extremely necessary to better understand the biology of the disease which may lead to the identification of novel prognostic biomarkers and efficient treatment options.

Cell cycle-specific transcription factor E2F1 was originally identified as a member of the E2F family of transcription factors, which consists of eight proteins commonly classified as activators (E2F1-E2F3a) or repressors (E2F3b-E2F8), based on their ability to either activate or repress target gene transcription (6). The activator E2F family proteins are required for cell proliferation, whereas the repressor E2Fs play a role in cell cycle exit and differentiation (7). A puzzling feature of the activator E2Fs, especially E2F1, is the ability to regulate the seemingly opposing processes of proliferation and apoptosis (8). Genome-wide analysis of targets of E2F1 using microarrays has also identified a large number of genes that are regulated by E2F1 in diverse cellular processes, such as replication, apoptosis, checkpoint control and DNA repair (9). In 
tumor biology, aberrant expression of E2F1 has been detected in various cancer cell lines and clinical tissues, including amplification of the E2F1 gene in erythroleukemia cell lines, elevated expression of E2F1 protein in breast cancer cell lines and head and neck carcinoma cell lines, and overexpression of E2F1 in invasive ductal breast cancer and non-small cell lung cancer, where high E2F1 expression associates with advanced disease and poor prognosis (10-14). Particularly, Ren et al (15) observed that E2F1 expression was elevated in advanced PCa and found that E2F1 knockdown could inhibit prostate tumor growth in vitro and in vivo through sensitizing tumor cells to ICAM-1 mediated anti-immunity by $\mathrm{NF}-\kappa \mathrm{B}$ modulation, highlighting the potential of E2F1 as a therapeutic target; Davis et al (16) indicated that elevated E2F1 expression, through its ability to repress androgen receptor (AR) transcription, might contribute to the progression of hormoneindependent PCa; Zheng et al (17) found that E2F1 could induce cancer cell survival via NF- $\mathrm{BB}$-dependent induction of EGR1 transcription in PCa cells; Libertini et al (18) described E2F1-dependent modifications of androgen dependence, differentiation, and sensitivity to apoptotic stimuli in PCa cell lines. It is, however, unclear whether E2F1 promotes metastasis of PCa cells and which of the E2F1 targets are key metastatic mediators in this cancer.

To address this problem, we determined the expression patterns of E2F1, at both mRNA and protein levels, in human PCa tissues, and evaluated the associations of E2F1 expression with clinicopathological characteristics and patient prognosis. Then, the roles of E2F1 on malignant phenotypes of PCa cells were investigated. Furthermore, the combined bioinformatic binding site prediction with chromatin immunoprecipitationPCR (ChIP-PCR) and western blot analysis was performed to identify the E2F1 targets in PCa cells.

\section{Materials and methods}

Patients and tissue samples. The present study was approved by the Research Ethics Committee of the Guangzhou First People's Hospital, Guangzhou Medical University, China. Informed consent was obtained from all the patients. The specimens were handled and made anonymous according to the ethical and legal standards.

Cell culture. Human PCa cell lines, LNCaP and PC3 were purchased from Guangzhou Land Biosciences Co., Ltd., (Guangzhou, China) and were cultured in RPMI-1640 medium (Cellgro, Manassas, VA, USA) supplemented with $10 \%$ fetal bovine serum (FBS; Cellgro), $2 \mathrm{mM}$ L-glutamine and antibiotics. All cell lines were maintained at $37^{\circ} \mathrm{C}$ in a humidified chamber supplemented with $5 \% \mathrm{CO}_{2}$.

Animals. Animal experiments in the present study were performed in compliance with the guidelines of the Institute for Laboratory Animal Research at Guangzhou Medical University (Guangzhou, China). A total of $8 \mathrm{BALB} / \mathrm{c}$ nude mice (6-8-week-old males) were purchased from Guangdong Medical Laboratory Animal Center and were housed 4 per cage in wire-top cages with sawdust bedding in an isolated, clean, air-conditioned room at a temperature of $25-26^{\circ} \mathrm{C}$ and a relative humidity of $\sim 50 \%$, lit $12 \mathrm{~h}$ /day.
Cell transfection. The siRNAs of E2F1 and control (cat nos. siB11715100401 and siN05815122147; Guangzhou RiboBio Co., Ltd., Guangzhou, China) were respectively transfected in LNCaP cells with Lipofectamine 2000. The expression of E2F1 was detected by qPCR and western blot analyis. The results showed that the siRNA with sequence (5'-CCUGAUGAA UAUCUGUACUdTdT-3') inhibited the E2F1 expression significantly and selected to construct the sh-E2F1 plasmid as an insert sequence. The pGreenPuro Scramble Hairpin Control as sh-RNA negative control was purchased from SBI (cat. no. MZIP000-PA-1; System Biosciences, Mountain View, CA, USA) and the shRNA-E2F1 was constructed using the pGreenPuro Scramble Hairpin Control as the bone plasmid. To make the virus, 293TN cells were transfected with shRNA-E2F1 or sh-RNA negative control with plasmid and Lipofectamine 2000, and then after 3 days, the virus particles were collected according to the packaging protocol of SBI with the Lenti-Concentin virus precipitation solution (cat. no. LV810A-1; System Biosciences). LNCaP and PC3 cells were infected by virus solution with TransDux virus transduction reagent (cat. no. LV850A-1; System Biosciences). The infected cells were detected by fluorescence microscope and isolated with a flow cytometer to construct the stable sh-E2F1 and control cell lines.

Western blot analysis. Proteins were extracted $48 \mathrm{~h}$ posttransfection for western blot analyses. Proteins $(40 \mu \mathrm{g})$ were fractioned on SDS-PAGE and transferred onto Hybond nitrocellulose membranes (GE Healthcare). The membranes were blocked with 5\% skim milk in PBS-Tween 20 and probed with anti-E2F1 (ab-64161; Abcam Co., Ltd., Hong Kong), anti-CD147 (ab-108308; Abcam) and anti-GAPDH (P30008; Abmart, Co., Ltd., Shanghai, China). The results were visualized with the SuperSignal West Pico chemiluminescent detection system (Pierce Biotechnology, Rockford, IL, USA). GAPDH was used as an internal loading control.

Immunohistochemistry. Expression pattern and subcellular localization of E2F1 protein in clinical PCa tissues were detected by immunohistochemistry and the immunoreactivity scores (IRS) were calculated according to the protocol of our previous studies $(19,20)$. IRS were scored by two independent experienced pathologists, who were blinded to the clinicopathological data and clinical outcomes of the patients. The number of positive-staining cells in ten representative microscopic fields was counted and the percentage of positive cells was calculated. Given the heterogeneity of the staining of the target genes or proteins, tumor specimens were scored in a semi-quantitative manner. The percentage was grouped as follows: 0 (0\%), 1 (1-10\%), $2(11-50 \%)$ and 3 (>50\%). The staining intensity was categoried as follows: 0 (negative), 1 (weak), 2 (moderate) and 3 (strong). A final score was obtained for each case by the sum of the percentages and the intensity scores. The antibodies used in this study were: E2F1 antibody (ab-64161; Abcam); CD147 antibody (ab-108308; Abcam); vimentin antibody (za-0511; ZSGB-BIO Co., Ltd., Beijing, China); E-cadherin antibody (za-0565; ZSGB-BIO).

Cell cycle analysis. The effects of E2F1 on cell cycle progression were determined by fluorescence-activated cell sorter 
(FACS) analysis using the Cell Cycle and Apoptosis Analysis kit (Beyotime Institute of Biotechnology, Shanghai, China) according to the manufacturer's instructions. Ten thousand cells were counted per sample. All experiments were done in triplicate. Mean normalized gene expression $\pm \mathrm{SE}$ was calculated from independent experiments.

Cell invasion assays. The Transwell inserts (8- $\mu \mathrm{m}$ pores) were filled with $50 \mu 1$ of a mixture of serum-free RPMI1640 medium (Cellgro) and Matrigel (1:10; BD Biosciences, San Jose, CA, USA). The inserts were then placed in 24-well tissue culture plates (Transwell; Corning Incorp., Corning, NY, USA) containing 10\% FBS-medium. After solidification by incubation in $37^{\circ} \mathrm{C}$ for $4 \mathrm{~h}, 5 \times 10^{4}$ cells in $200 \mu \mathrm{l}$ medium were placed in upper chambers. Following $48 \mathrm{~h}$ of incubation at $37^{\circ}$ with $5 \% \mathrm{CO}_{2}$ and in culture medium with mitomycin to stop the mitosis, the membranes were fixed with $10 \%$ formalin and stained with $0.05 \%$ crystal violet. The number of cells that migrated through the pores was assessed and the data were expressed as mean \pm SD of three independent experiments.

Migration assays. For the scratch wound-healing motility assay, a scratch was made with a pipette tip when the cells reach confluence. After being cultured under standard conditions with mitomycin for $24 \mathrm{~h}$, plates were washed twice with fresh medium to remove non-adherent cells and then photographed. The cell migrated from the wound edge were counted and the data were expressed as the mean \pm SD of three independent experiments.

Generation of the in vivo xenograft model. For the in vivo tumor formation assays, $\mathrm{PC} 3$ or LNCaP cells transfected with shRNA-E2F1 were trypsinized and suspended in phosphate-buffered saline (PBS). Then, the cells were subcutaneously injected into the flanks of each nude mouse (4 per group). PC 3 cells were subcutaneously injected with $0.1 \mathrm{ml}$ volume of $1 \times 10^{6}$ cells. LNCaP cells were subcutaneously injected at a $0.1 \mathrm{ml}$ mixture of $1 \times 10^{6}$ cells and an $0.05 \mathrm{ml}$ Matrigel (cat. no. 356234; BD Biosciences), reaching a total concentration of $10 \mathrm{mg} / \mathrm{ml}$. The tumor sizes were measured at 4-day intervals, as soon as the tumors were measurable and the tumor volumes were calculated: $\mathrm{V}\left(\mathrm{mm}^{3}\right)$ $=\operatorname{width}^{2}\left(\mathrm{~mm}^{2}\right) \times$ length $(\mathrm{mm}) / 2$. On day 44 for $\mathrm{LNCaP}$ and day 36 for PC 3 groups, the mice were sacrificed. The mice were manipulated and housed according to the protocols approved by the Institute for Laboratory Animal Research at Guangzhou Medical University.

Bioinformatics prediction of E2F1 binding sites in sets of genes. Three online bioinformatics software including oPOSSUM (http://www.cisreg.ca/oPOSSUM/, Last updated: Jan 31st, 2007) (21), ConSite (http://consite.genereg.net/) and PROMO (version 3.0.2) (22) were used to predict E2F1 binding sites in sets of genes.

CHIP-PCR. ChIP was conducted with Chromatin immunoprecipitation assay kit (cat. no. 26156; Thermo Fisher Scientific, Waltham, MA, USA) according to the manufacturer's instructions. In brief, cells were fixed in $1 \%$ formaldehyde at room temperature for $10 \mathrm{~min}$; the nuclei were isolated with nuclear lysis buffer (Thermo Fisher Scientific) supplemented with protease inhibitor cocktail (Thermo Fisher Scientific). Chromatin DNA was sonicated and sheared to a length between 200 and $500 \mathrm{bp}$. The sheared chromatin was immunoprecipitated at $4^{\circ} \mathrm{C}$ overnight with anti-E2F1 (Cell Signaling Technology). Normal rabbit IgG was used as a negative control and genome DNA was used as an input control. Protein/DNA complex was reverse cross-linked and DNA was purified using spin columns. Purified DNA was detected with quantitative PCR. The sequences used in the present study were: E2F1/ CD147 F1, 5'-ATGTGCGTCTCGCCATGTT-3'; E2F1/CD147 R1, 5'-TGTGGCTCACGTCTGTACTC-3'.

Statistical analysis. The version 13.0 SPSS for Windows (SPSS, Inc., IL, USA) and SAS 9.1 (SAS Institute, Cary, NC, USA) software was used for statistical analysis. Continuous variables were expressed as mean $\pm \mathrm{SD}$. Statistical analyses of PCR and western blot analysis were conducted using Wilcoxon signed-rank test. Statistical analysis was performed independently by two biostatisticians with Fisher's exact test for any $2 \times 2$ tables and Pearson $\chi^{2}$ test for non- $2 \times 2$ tables. Kaplan-Meier method was used for the survival analyses. The Pearson's correlation was calculated between the expression levels of E2F1 and CD147 in PCa tissues. Differences were considered statistically significant when the P-value was $<0.05$.

\section{Results}

Overexpression of E2F1 in human PCa tissues. Expression pattern and sub-cellular localization of E2F1 protein in 97 $\mathrm{PCa}$ and 81 adjacent non-cancerous prostate tissues were detected by immunohistochemistry. As shown in Fig. 1A-C, E2F1 protein immunostaining occurred mainly in the cytoplasm and nuclei of PCa cells, but weakly or negatively in adjacent non-cancerous prostate tissues. Statistical analysis found that the expression levels of E2F1 protein in PCa tissues were significantly higher than those in adjacent non-cancerous prostate tissues (IRS, PCa, $6.60 \pm 0.76$ vs. Benign, $5.67 \pm 1.18$, $\mathrm{P}<0.001$; Fig. 1D).

Overexpression of E2F1 associates with the aggressive progression of human PCa. Table I summarizes the associations between E2F1 protein expression and various clinicopathological features of PCa patients. We found that E2F1 protein overexpression in PCa tissues was significantly associated with high Gleason score $(\mathrm{P}=0.005)$ and advanced pathological stage $(\mathrm{P}=0.005)$, which was consistent with the statistical results of E2F1 mRNA expression based on Taylor dataset (Gleason score $\geq 8$ vs. Gleason score $<8, \mathrm{P}=0.013$; pathological stage $>\mathrm{T} 3 \mathrm{~A}$ vs. pathological stage $<\mathrm{T} 3 \mathrm{~A}, \mathrm{P}=0.002$ ). According to the Taylor dataset, we also found that $\mathrm{PCa}$ patients with positive metastasis and short overall survival time had higher expression levels of E2F1 mRNA expression than those with negative metastasis $(\mathrm{P}<0.001)$ and long overall survival time ( $\mathrm{P}=0.003)$.

Overexpression of E2F1 predicts poor prognosis of human $\mathrm{PC} a$. To evaluate the prognostic value of E2F1 expression in 
A

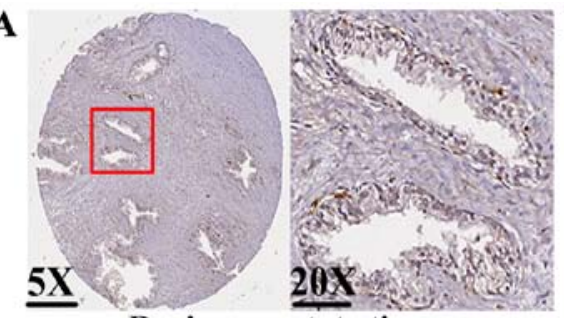

Benign prostate tissue

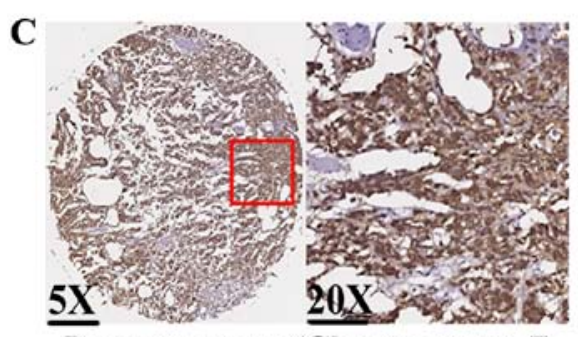

Prostate cancer(Gleason score $>7$ )

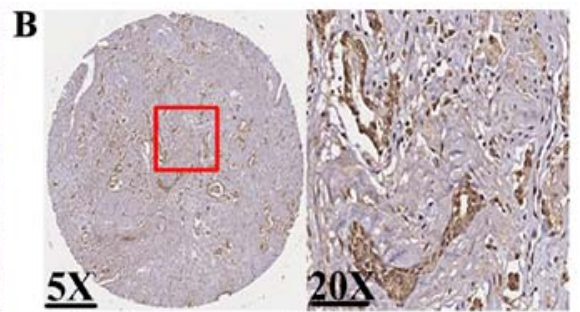

Prostate cancer(Gleason score $=7$ )

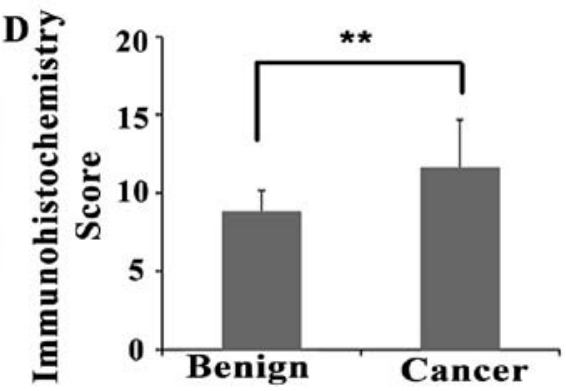

Figure 1. Immunohistochemical staining for E2F1 protein in human prostate cancer (PCa) and adjacent non-cancerous prostate tissues (original magnification, $\mathrm{x} 20$ ). (A) E2F1 protein was weakly or negatively expressed in normal prostate gland cells in adjacent non-cancerous prostate tissues; (B and C) E2F1 protein immunostaining occurred mainly in the cytoplasm of cancer cells in PCa tissues; (D) The expression levels of E2F1 protein in PCa tissues were significantly higher than those in adjacent non-cancerous prostate tissues (IRS: $\mathrm{PCa}=6.60 \pm 0.76$ vs. $\mathrm{Benign}=5.67 \pm 1.18 ; \mathrm{P}<0.001$ ).

Table I. Associations of E2F1 protein and mRNA expression with various clinicopathological characteristics of human prostate cancer $(\mathrm{PCa})$.

\begin{tabular}{|c|c|c|c|c|c|c|}
\hline \multirow[b]{2}{*}{ Clinical features } & \multicolumn{3}{|c|}{ E2F1 in our tissue microarray } & \multicolumn{3}{|c|}{ E2F1 expression in Taylor dataset } \\
\hline & No. of cases & Mean \pm SD & P-value & No. of cases & Mean \pm SD & P-value \\
\hline \multicolumn{7}{|l|}{ Age (years) } \\
\hline$<70$ & 43 & $6.47 \pm 0.94$ & \multirow[t]{2}{*}{0.162} & 144 & $7.59 \pm 0.20$ & \multirow[t]{2}{*}{0.770} \\
\hline$\geq 70$ & 54 & $6.70 \pm 0.57$ & & 6 & $7.61 \pm 0.13$ & \\
\hline \multicolumn{7}{|c|}{ Serum PSA (ng/ml) } \\
\hline$<4$ & - & - & \multirow[t]{2}{*}{-} & 24 & $7.59 \pm 0.21$ & \multirow[t]{2}{*}{0.212} \\
\hline$\geq 4$ & - & - & & 123 & $7.58 \pm 0.19$ & \\
\hline \multicolumn{7}{|l|}{ Gleason score } \\
\hline$<8$ & 70 & $6.50 \pm 0.85$ & \multirow[t]{2}{*}{0.005} & 117 & $7.56 \pm 0.25$ & \multirow[t]{2}{*}{0.013} \\
\hline$\geq 8$ & 27 & $8.85 \pm 0.36$ & & 22 & $7.68 \pm 0.25$ & \\
\hline \multicolumn{7}{|l|}{ Pathological stage } \\
\hline$<\mathrm{T} 3 \mathrm{~A}$ & 70 & $6.50 \pm 0.85$ & \multirow[t]{2}{*}{0.005} & 86 & $7.54 \pm 0.18$ & \multirow[t]{2}{*}{0.002} \\
\hline$\geq \mathrm{T} 3 \mathrm{~A}$ & 27 & $8.85 \pm 0.36$ & & 55 & $7.64 \pm 0.20$ & \\
\hline \multicolumn{7}{|l|}{ Metastasis } \\
\hline No & 97 & $6.60 \pm 0.76$ & \multirow[t]{2}{*}{-} & 122 & $7.56 \pm 0.18$ & \multirow[t]{2}{*}{$<0.001$} \\
\hline Yes & 0 & - & & 28 & $7.74 \pm 0.22$ & \\
\hline \multicolumn{7}{|l|}{ Overall survival } \\
\hline Alive & - & - & \multirow[t]{2}{*}{-} & 131 & $7.57 \pm 0.18$ & \multirow[t]{2}{*}{0.003} \\
\hline Die & - & - & & 19 & $7.71 \pm 0.28$ & \\
\hline \multicolumn{7}{|l|}{ PSA failure } \\
\hline Negative & - & - & \multirow[t]{2}{*}{-} & 104 & $7.56 \pm 0.18$ & \multirow[t]{2}{*}{0.064} \\
\hline Positive & - & - & & 36 & $7.63 \pm 1.18$ & \\
\hline
\end{tabular}

$\mathrm{PCa}$, the Kaplan-Meier method was performed to analyze the correlation of E2F1 expression with biochemical recurrence
(BCR)-free survival and overall survival of PCa patients based on Taylor dataset. The median value of E2F1 mRNA 

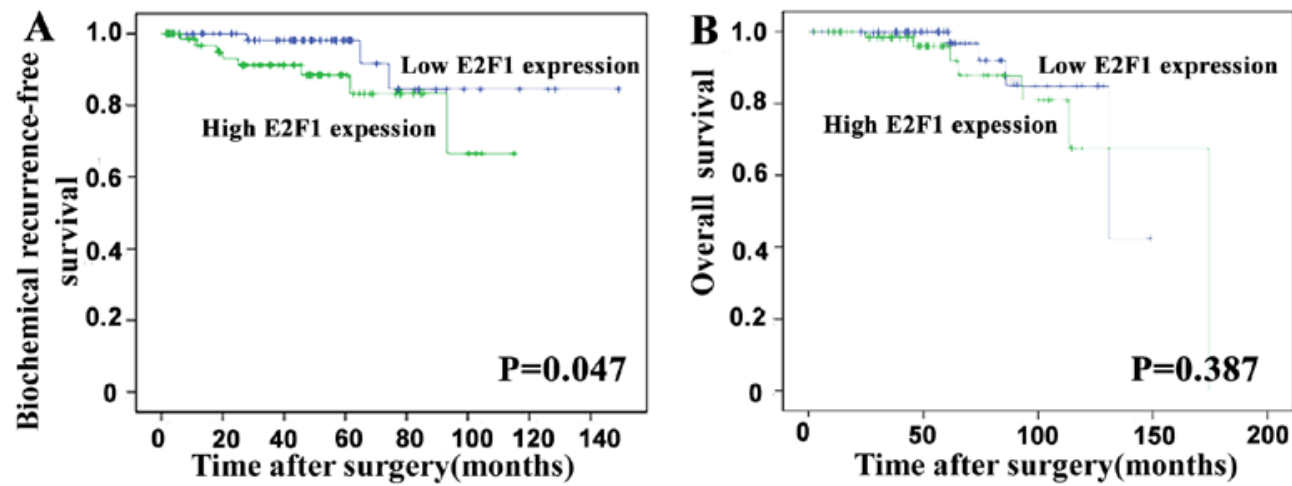

Figure 2. Kaplan-Meier survival curves for E2F1 expression in human prostate cancer (PCa). (A) The biochemical recurrence-free survival of PCa patients with high E2F1 expression was significantly shorter than those with low E2F1 expression ( $\mathrm{P}=0.047)$; (B) the overall survival of PCa patients with high E2F1 expression is not statistically significant compared to low $\mathrm{E} 2 \mathrm{~F} 1$ expression $(\mathrm{P}=0.387)$.

A

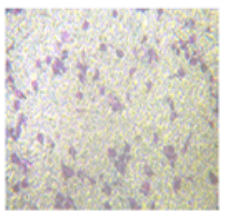

sh-E2F1

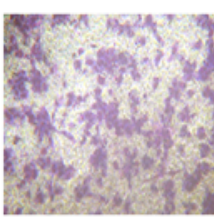

Vector

LNCaP

B $0 \mathrm{~h}$

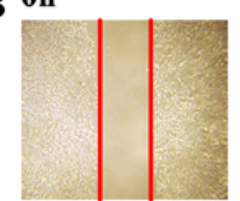

24h

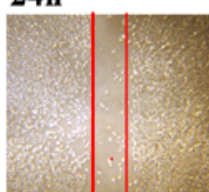

sh-E2F1
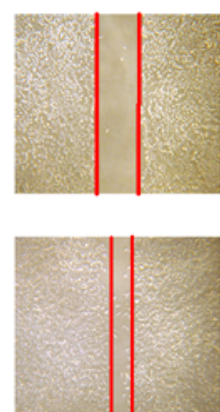

Vector LNCaP

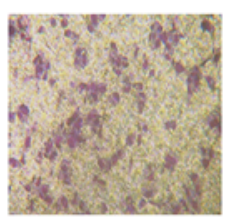

sh-E2F1

PC3
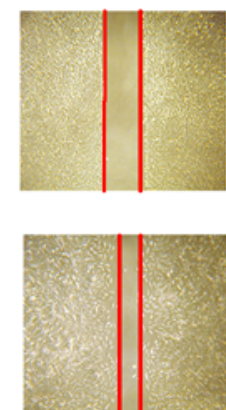

Sh-E2F1

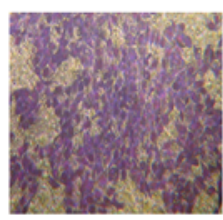

Vector
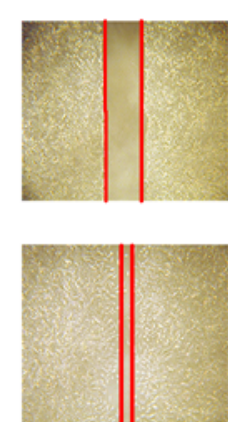

PC3
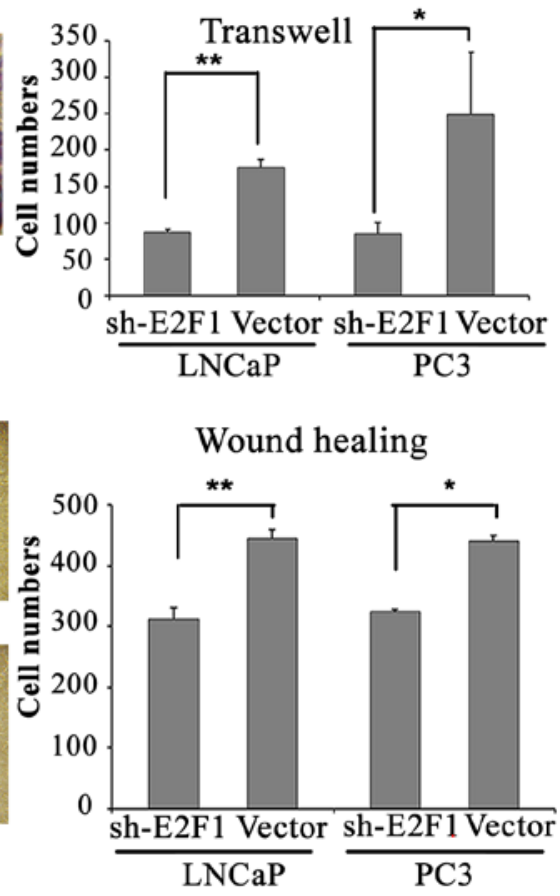

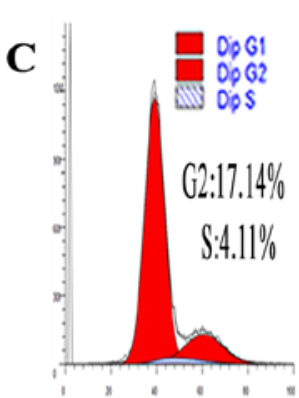

sh-E2F1

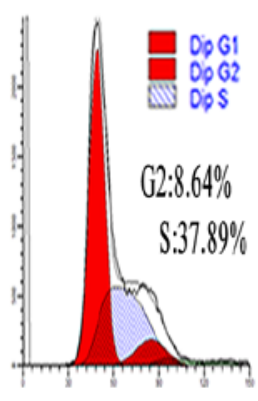

LNCaP

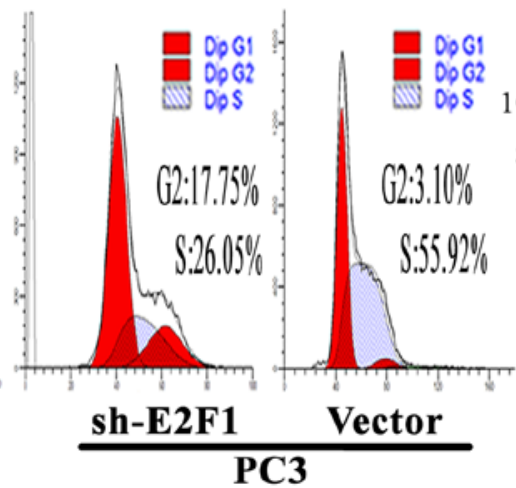

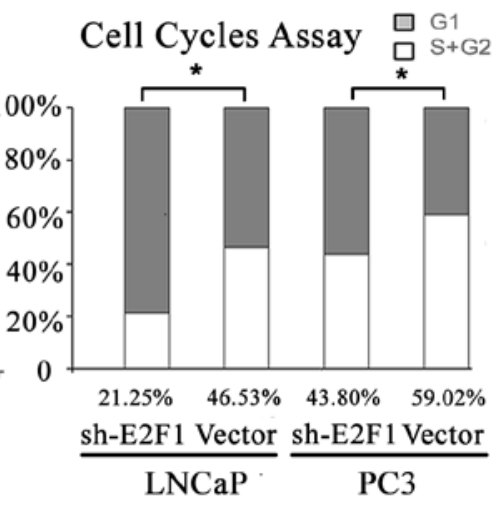

Figure 3. Knock-down of E2F1 inhibits cell cycle progression, invasion and migration of PCa cell lines in vitro. (A and B) The migration and invasive activities of both PC3 and LNCaP cells were inhibited by the downregulating of E2F1 expression. 'vector', scramble shRNA as negative control; sh-E2F1, shRNA for $\mathrm{E} 2 \mathrm{~F} 1$; (C) the knockdown of E2F1 could lead to substantial accumulation of the cell population at the G1 stage of the cell cycle in both PC3 and LNCaP cells $\left(\mathrm{P}=0.015\right.$ and 0.010 , respectively); data are expressed as mean $\pm \mathrm{SD}$. ${ }^{*} \mathrm{P}<0.05$. ${ }^{* *} \mathrm{P}<0.01$.

expression in all PCa tissues was used as the cut-off point to divide all cases into high $(n=75)$ and low $(n=74)$ E2F1 expression groups. Pairwise comparisons showed significant differences in the BCR-free survival ( $\mathrm{P}=0.047$; Fig. 2A) 


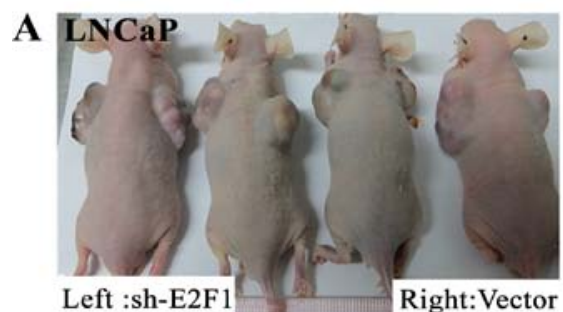

C
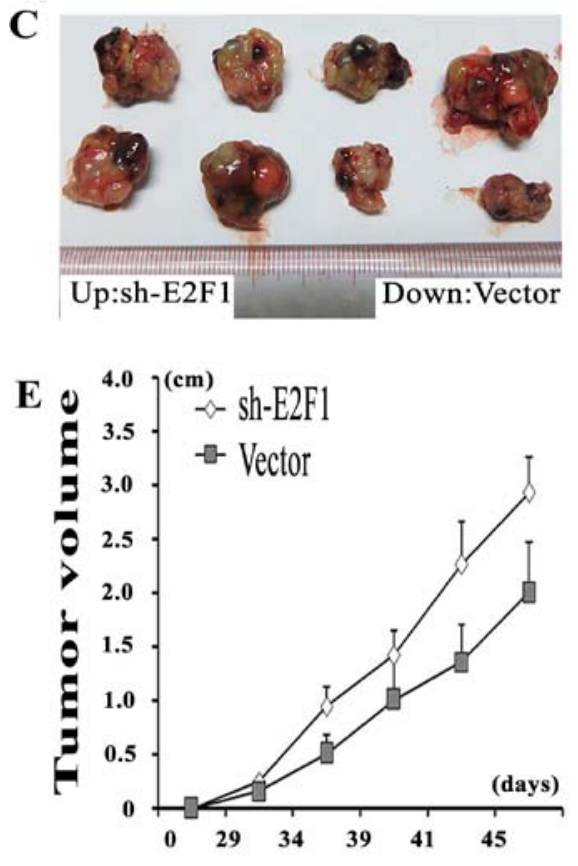
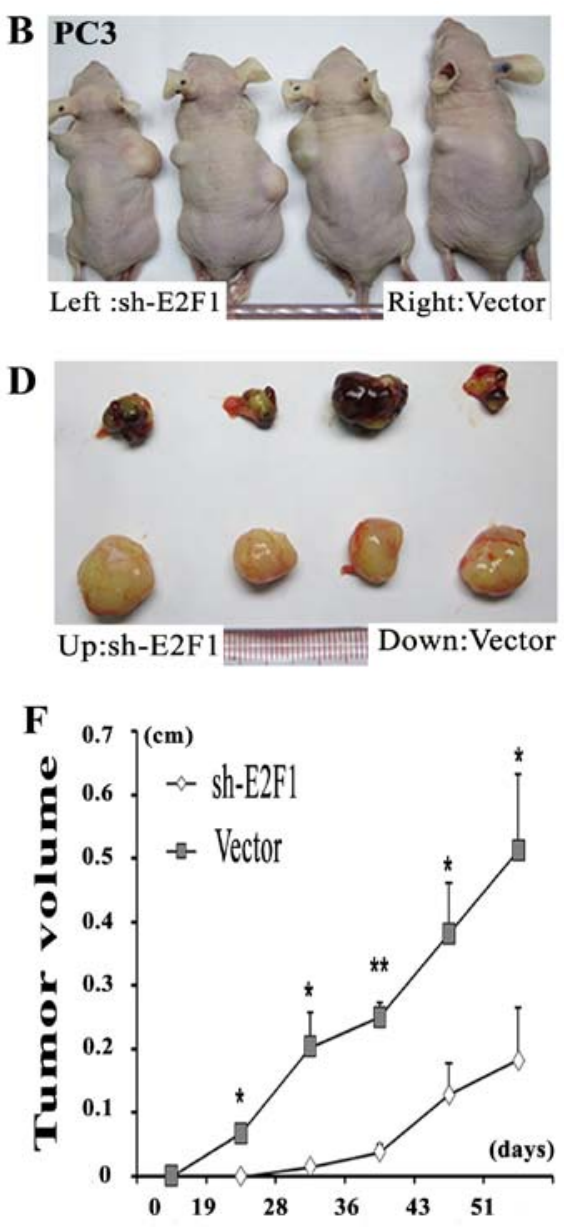

Figure 4. Knockdown of E2F1 suppresses tumor growth in vivo. The PC3 cells with the knockdown of E2F1 formed significantly smaller tumor nodules and remarkably slowed tumor xenograft growth compared with the controls. 'vector', scramble shRNA as negative control; sh-E2F1, shRNA for E2F1. Data are expressed as mean $\pm \mathrm{SD}$. ${ }^{*} \mathrm{P}<0.05 ;{ }^{* *} \mathrm{P}<0.01$.

between patients with high and low E2F1 expression groups, except the overall survival ( $\mathrm{P}=0.387$; Fig. $2 \mathrm{~B})$.

Knock-down of E2F1 inhibits cell cycle progression, invasion and migration of $P C a$ cell lines in vitro. To determine the oncogenic roles of $\mathrm{E} 2 \mathrm{~F} 1$ in $\mathrm{PCa}$, we detected the cell cycle progression, invasion and migration abilities of $\mathrm{PC} 3$ and LNCaP cells with the knock-down of E2F1. According to the western blot analysis, the expression of E2F1 protein was successfully suppressed by sh-E2F1 vector in both PC3 and LNCaP cells (Fig. 6B). Then, we found that the knockdown of E2F1 could lead to substantial accumulation of the cell population at the G1 stage of the cell cycle in PC3 and LNCaP cells $(\mathrm{P}=0.015$ and $\mathrm{P}=0.010$, respectively; Fig. $3 \mathrm{C})$. Moreover, the invasive and migration activities of both $\mathrm{PC} 3$ and $\mathrm{LNCaP}$ cells were inhibited by the downregulating of E2F1 expression (all $\mathrm{P}<0.05$; Fig. 3A and B).

Knockdown of E2F1 suppresses tumor growth and invasion in vivo. To better evaluate the biological functions of $\mathrm{E} 2 \mathrm{~F} 1$ in vivo, $\mathrm{LNCaP}$ and $\mathrm{PC} 3$ cell lines transfected with sh-E2F1 were used and subcutaneously injected into the flank of each male nude mouse, simultaneously, the vector control PCa cells were subcutaneously injected into the other flank of the same mouse. The PC3 cells with the knockdown of E2F1 formed significantly smaller tumor nodules and remarkably slowed tumor xenograft growth compared with the controls (Fig. 4B, $\mathrm{D}$ and $\mathrm{F}$ ). However, there is no significantly difference between the tumor size of E2F1 knockdown group and the control group in LNCaP cells (Fig. 4A, C and E).

Next, immunohistochemical analysis was performed to detect the expression patterns of epithelial marker E-cadherin and mesenchymal marker vimentin in the tumor xenografts, in order to evaluate the tumor invasive tendency in different groups. The results indicated that the expression level of vimentin protein in the tumor xenografts established by $\mathrm{LNCaP}$ or PC3 cells transfected with sh-E2F1 was remarkably lower than that in the xenografts established by cells transfected with control vectors (Fig. 5A). In contrast, the tumor xenografts established by LNCaP or PC3 cells with low E2F1 expression presented significantly more E-cadherin protein than the control xenografts (Fig. 5B). These results strongly demonstrated that the knockdown of E2F1 could significantly inhibit tumor growth and invasion in vivo.

Positive correlation between expression of E2F1 and CD147. In a previous study, we found that CD147 expression in human PCa tissues was significantly higher than those in adjacent non-cancerous prostate tissues and might be an important factor in predicting the prognosis of the PCa patients (23). 
A

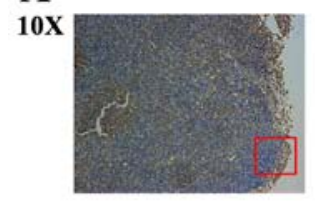

$40 X$

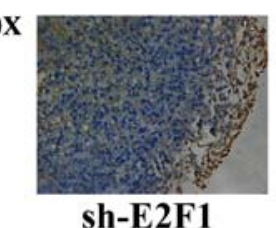

Vimentin expression
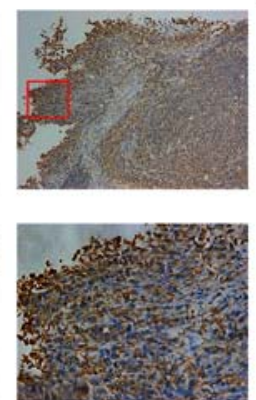

Vector

LNCaP
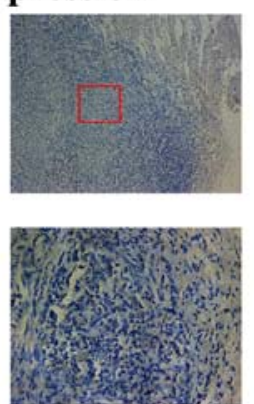

sh-E2F1
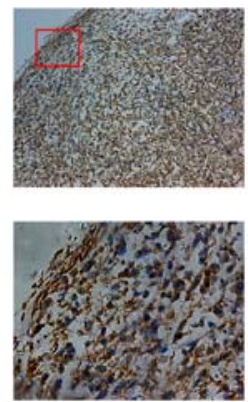

Vector

PC3

$\mathbf{B}$

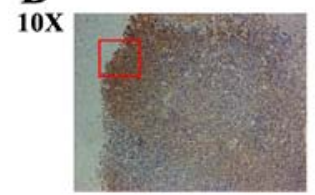

40X

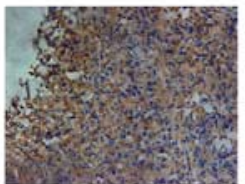

sh-E2F1
E-cadherin expression
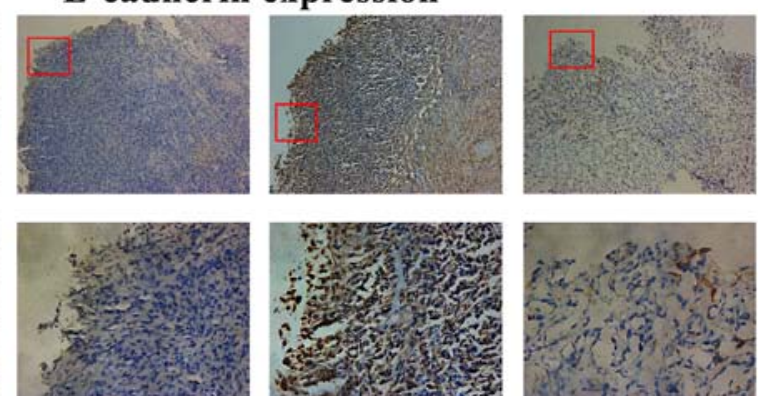

sh-E2F1

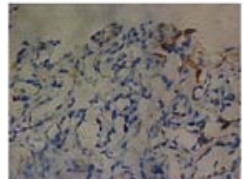

Vector

LNCaP

PC3

Figure 5. Knockdown of E2F1 suppresses tumor growth and invasion in vivo. (A) Expression level of vimentin protein in the tumor xenografts established with LNCaP or PC 3 cells transfected with sh-E2F1 was remarkably lower than that in the xenografts established by cells transfected with control vectors; (B) The tumor xenografts established with LNCaP or PC3 cells with low E2F1 expression presented significantly more E-cadherin protein than the control xenografts. 'vector', scramble shRNA as negative control.

Based on the Taylor dataset, the Pearson correlation analysis clearly showed a positive correlation between CD147 and E2F1 mRNA expression in PCa tissues $(r=0.225, \mathrm{P}=0.006$; Fig. 6A). To identify E2F1 target genes in the human genome, 3 online bioinformatics software including oPOSSUM, ConSite and PROMO were used and commonly predicted CD147 as a potential target of E2F1. As shown in Fig. 6C, E2F1 and CD147 could directly bind to the putative E2F1-CD147 binding region (sequence: GTTGCGG). To validate this prediction, we detected the expression of CD147 at protein level in $2 \mathrm{PCa}$ cell lines (PC3 and LNCAP) transfected with sh-E2F1. As shown in Fig. 6B, knocking down E2F1 resulted in significant downregulation of CD147 protein expression. Thus, we speculated that E2F1 might upregulate CD147 expression in PC3 and LNCAP cells. To confirm our speculation, we analyzed the binding site of CD147 promoter by ChIP-PCR. Compared to the negative control group, E2F1 could directly bind to a putative site in the promoter of CD147 (Fig. 6D). Also, the tumor xenografts established by LNCaP or PC3 cells with low E2F1 expression significantly suppressed the expression of CD147 compared to the control xenografts (Fig. 6E).

\section{Discussion}

The transcription factor E2F1 expression has been detected in several human cancers, and oncogenic and tumor-suppressive roles have been proposed for E2F1 depending on the cancer type and, in some cases, the subtype. For example, Li et al (24) demonstrated the increased expression of E2F1 in small cell lung cancer (SCLC), and confirmed that E2F1 could directly enhance matrix metalloproteinase (MMP) transcription by binding to the E2F1 binding sequences in the promoter, or indirectly activate MMPs through enhanced Sp1 and NF- $\kappa B$ as a consequence of E2F1 activation in SCLC; Ma et al (25) indicated that the overexpression of E2F1 might promote tumor malignancy and correlates with TNM stages in clear cell renal cell carcinoma; Engelmann et al (26) also revealed that E2F1 might function as an enhancer of angiogenesis via regulation of VEGF-C/VEGFR-3 signaling in tumors to cooperatively activate PDGF-B expression, and targeting this pathway might be reasonable to complement standard anti-angiogenic treatment of cancers with deregulated E2F1; on the contrary, E2F1 has been revealed to be a mediator of apoptosis, and in clinical trials where its high expression leads to improved survival of patients with adjuvant chemoradiation therapy (27). These findings suggest that E2F1 exhibits dual properties, acting as a tumor suppressor and oncogene.

Previous studies have reported the oncogenic role of E2F1 in the control of PCa cell cycle, and the regulation of proliferation and apoptosis (15-18). However, its clinical significance in PCa remains unclear. In the present study, our clinical evidence revealed that the upregulation of E2F1 was remarkably associated with the tumor aggressive clinicopathological 
A

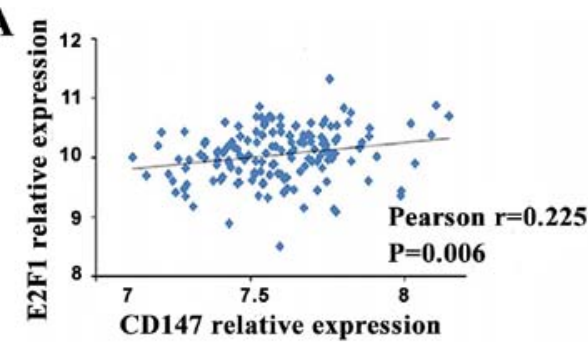

C

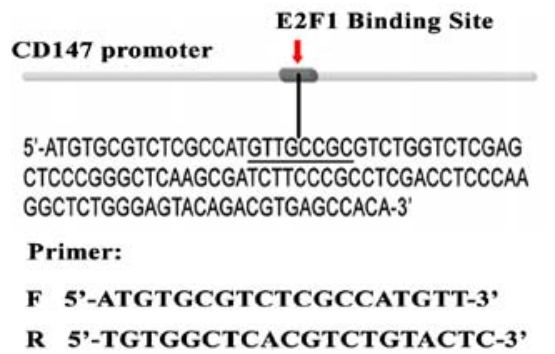

$\mathbf{E}$

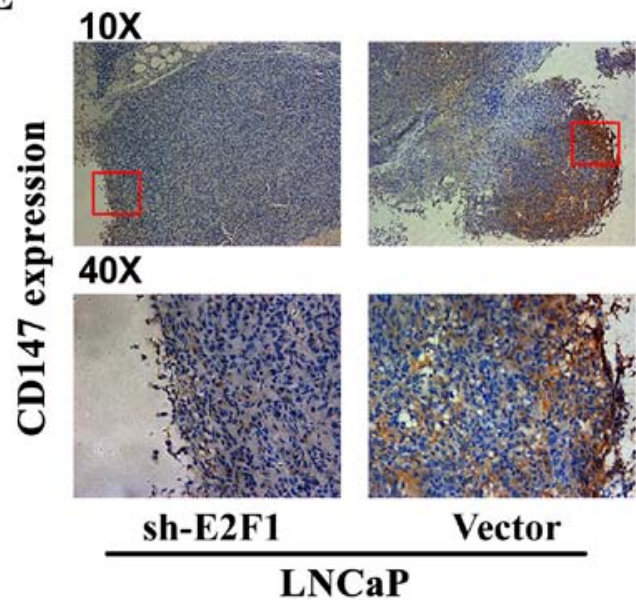

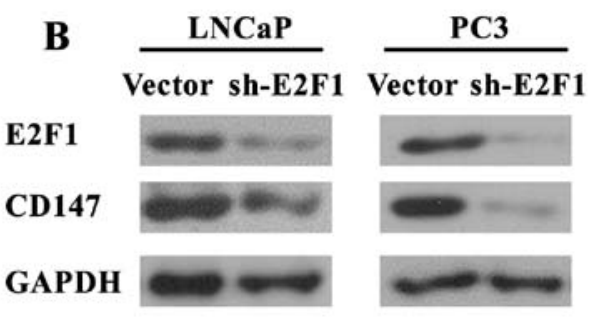

D
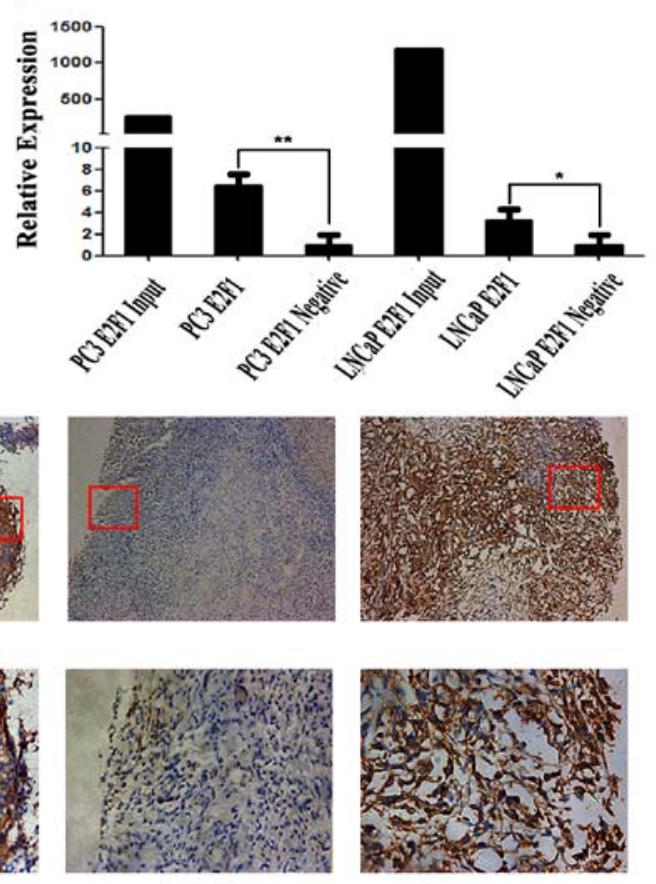

sh-E2F1

Vector

PC3

Figure 6. Positive correlation between expression of E2F1 and CD147. (A) Based on the Taylor dataset, Pearson's correlation analysis clearly showed a positive correlation between CD147 and E2F1 mRNA expression in PCa tissues $(\mathrm{r}=0.225, \mathrm{P}=0.006)$; (B) western blot analysis showed that the expression of E2F1 and CD147 protein was successfully suppressed by sh-E2F1 vector in both PC3 and LNCaP cells; (C) putative E2F1-CD147 binding region (sequence: GTTGCCGC) predicted by the online bioinformatics software including oPOSSUM, ConSite and PROMO; (D) ChIP-PCR analysis. Compared to the negative control group, E2F1 could directly bind to a putative site in the promoter of CD147. 'vector', scramble shRNA as negative control; sh-E2F1, shRNA for E2F1. Data were expressed as mean $\pm \mathrm{SD}$. ${ }^{*} \mathrm{P}<0.05 ;{ }^{* *} \mathrm{P}<0.01$. (E) expression level of CD147 protein in the tumor xenografts established by LNCaP or PC 3 cells transfected with sh-E2F1 was remarkably lower than that in the xenografts established by cells transfected with control vectors.

features and might act as an important prognostic factor for BCR-free survivals in patients with $\mathrm{PCa}$, which prompted us to determine the roles of E2F1 in malignant phenotypes of $\mathrm{PCa}$ in vitro and in vivo models. Our data showed that the downregulation of E2F1 expression by shRNA-E2F1 could suppress $\mathrm{PCa}$ cell cycle progression, invasion and migration in vitro, and caused a dramatic decrease in PC3 tumor growth and suppressed tumor invasion in vivo. These findings delineate an as yet unrecognized function of E2F1 as enhancer of tumor invasion and migration of $\mathrm{PCa}$.

Furthermore, we determined that E2F1 could directly target CD147 and be implicated in carcinogenesis of PCa through the regulation of CD147, which is a transmembrane glycoprotein, and member of the immunoglobulin superfamily molecules expressed on the cell surface of most tumor cells (28). Functionally, CD147 may exert an oncogenic role by stimulating the synthesis of several MMPs, activating tumor angiogenesis and cell survival signaling (29). Our previous studies have reported the involvement of CD147 in human PCa. In 2008, we found that CD147, MMP-1, MMP-2 and MMP-9 expression levels were all significantly higher in PCa tissues than in benign tissues, and there was a significant positive correlation between CD147 and MMP-1, MMP-2 and MMP-9 expression in PCa tissues (30). Then, we identified CD147 as an independent prognostic factor for patients with PCa (31). Moreover, in 2012, we examined whether the expression of CD147 can be used as a prognostic marker for predicting PCa progression based on a large cohort of clinical cases. As a result, our data revealed that the increased expression of CD147 was correlated with higher Gleason scores, positive surgical margin, prostate-specific antigen failure, metastasis and reduced overall survival. Importantly, higher expression of CD147 can serve as an independent prognostic predictor for PSA failure-free survival in PCa patients when they are stratified by Gleason scores (31). In the present study, we confirmed that CD147 might function as a direct target for E2F1 in PCa 
cells through bioinformatics binding site prediction, combined with ChIP-PCR, and western blot analysis. The expression of CD147 mRNA in PCa tissues was positively correlated with that of E2F1 mRNA. Then, knocking down the expression of E2F1 was able to suppress the expression of CD147 in PCa cells in vitro and in vivo. Given that $\mathrm{CD} 147$ has been reported to be significantly upregulated in advanced $\mathrm{PCa}$ and could promote cell migration and invasion, the oncogenic role of $\mathrm{E} 2 \mathrm{~F} 1$ in $\mathrm{PCa}$ might be exerted by regulating CD147 expression.

In conclusion, E2F1 may promote tumor cell invasion and migration through the regulation of CD147 in PCa cells. Importantly, E2F1 may function as a biomarker that can differentiate patients with biochemical recurrent and nonbiochemical recurrent disease following RP, highlighting its potential as a therapeutic target.

\section{Acknowledgements}

The present study was supported by grants from the National Natural Science Foundation of China (81170699 and 81272813), the Natural Science Foundation of Guangdong Province (2014A030310501) and the Science and Technology Project of Bureau of Health in Guangzhou Municipality (20141A011006).

\section{References}

1. Siegel R, Naishadham D and Jemal A: Cancer statistics, 2013. CA Cancer J Clin 63: 11-30, 2013.

2. Nandana S and Chung LW: Prostate cancer progression and metastasis: Potential regulatory pathways for therapeutic targeting. Am J Clin Exp Urol 2: 92-101, 2014.

3. Han M, Partin AW, Zahurak M, Piantadosi S, Epstein JI and Walsh PC: Biochemical (prostate specific antigen) recurrence probability following radical prostatectomy for clinically localized prostate cancer. J Urol 169: 517-523, 2003.

4. Lapointe J,Li C, Higgins JP, van de Rijn M, BairE, Montgomery K, Ferrari M, Egevad L, Rayford W, Bergerheim U, et al: Gene expression profiling identifies clinically relevant subtypes of prostate cancer. Proc Natl Acad Sci USA 101: 811-816, 2004.

5. D'Amico AV, Moul J, Carroll PR, Sun L, Lubeck D and Chen MH: Cancer-specific mortality after surgery or radiation for patients with clinically localized prostate cancer managed during the prostate-specific antigen era. J Clin Oncol 21: 2163-2172, 2003.

6. Ogawa H, Ishiguro K, Gaubatz S, Livingston DM and Nakatani Y: A complex with chromatin modifiers that occupies E2F- and Myc-responsive genes in G0 cells. Science 296: 1132-1136, 2002

7. Ishida S, Huang E, Zuzan H, Spang R, Leone G, West M and Nevins JR: Role for E2F in control of both DNA replication and mitotic functions as revealed from DNA microarray analysis. Mol Cell Biol 21: 4684-4699, 2001.

8. Ginsberg D: E2F1 pathways to apoptosis. FEBS Lett 529: $122-125,2002$

9. Johnson DG: The paradox of E2F1: Oncogene and tumor suppressor gene. Mol Carcinog 27: 151-157, 2000.

10. Saito M, Helin K, Valentine MB, Griffith BB, Willman CL, Harlow E and Look AT: Amplification of the E2F1 transcription factor gene in the HEL erythroleukemia cell line. Genomics 25: $130-138,1995$.

11. Montenegro MF, Collado-González MM, Fernández-Pérez MP, Hammouda MB, Tolordava L, Gamkrelidze $M$ and Rodríguez-López JN: Promoting E2F1-mediated apoptosis in oestrogen receptor- $\alpha$-negative breast cancer cells. BMC Cancer 14: 539, 2014.

12. Lu M, Liu Z, Yu H, Wang LE, Li G, Sturgis EM, Johnson DG and Wei Q: Combined effects of E2F1 and E2F2 polymorphisms on risk and early onset of squamous cell carcinoma of the head and neck. Mol Carcinog 51 (Suppl 1): E132-E141, 2012.

13. Rizwani W, Schaal C, Kunigal S, Coppola D and Chellappan S: Mammalian lysine histone demethylase KDM2A regulates E2F1-mediated gene transcription in breast cancer cells. PLoS One 9: e100888, 2014
14. Hung JJ, Hsueh CT, Chen KH, Hsu WH and Wu YC: Clinical significance of E2F1 protein expression in non-small cell lung cancer. Exp Hematol Oncol 1: 18, 2012.

15. Ren Z, Kang W, Wang L, Sun B, Ma J, Zheng C, Sun J, Tian Z, Yang $X$ and Xiao W: E2F1 renders prostate cancer cell resistant to ICAM-1 mediated antitumor immunity by NF- $\kappa \mathrm{B}$ modulation. Mol Cancer 13: 84, 2014.

16. Davis JN, Wojno KJ, Daignault S, Hofer MD, Kuefer R, Rubin MA and Day ML: Elevated E2F1 inhibits transcription of the androgen receptor in metastatic hormone-resistant prostate cancer. Cancer Res 66: 11897-11906, 2006.

17. Zheng C, Ren Z, Wang H, Zhang W, Kalvakolanu DV, Tian Z and Xiao W: E2F1 Induces tumor cell survival via nuclear factorkappaB-dependent induction of EGR1 transcription in prostate cancer cells. Cancer Res 69: 2324-2331, 2009.

18. Libertini SJ, Tepper CG, Guadalupe M, Lu Y, Asmuth DM and Mudryj M: E2F1 expression in LNCaP prostate cancer cells deregulates androgen dependent growth, suppresses differentiation, and enhances apoptosis. Prostate 66: 70-81, 2006.

19. Lin ZY, Huang YQ, Zhang YQ, Han ZD, He HC, Ling XH, Fu X, Dai QS, Cai C, Chen JH, et al: MicroRNA-224 inhibits progression of human prostate cancer by downregulating TRIB1. Int J Cancer 135: 541-550, 2014.

20. Chen G, Liang YX, Zhu JG, Fu X, Chen YF, Mo RJ, Zhou L, $\mathrm{Fu} \mathrm{H}, \mathrm{Bi} \mathrm{XC}$, He HC, et al: CC chemokine ligand 18 correlates with malignant progression of prostate cancer. Biomed Res Int 2014: 230183, 2014.

21. Kwon AT, Arenillas DJ, Worsley Hunt R and Wasserman WW: oPOSSUM-3: Advanced analysis of regulatory motif overrepresentation across genes or ChIP-Seq datasets. G3 (Bethesda) 2: 987-1002, 2012.

22. Farré D, Roset R, Huerta M, Adsuara JE, Roselló L, Albà MM and Messeguer X: Identification of patterns in biological sequences at the ALGGEN server: PROMO and MALGEN. Nucleic Acids Res 31: 3651-3653, 2003.

23. Zhong WD, Liang YX, Lin SX, Li L, He HC, Bi XC, Han ZD, Dai QS, Ye YK, Chen QB, et al: Expression of CD147 is associated with prostate cancer progression. Int J Cancer 130: 300-308, 2012

24. Li Z, Guo Y, Jiang H, Zhang T, Jin C, Young CY and Yuan H: Differential regulation of MMPs by E2F1, Sp1 and NF-kappa B controls the small cell lung cancer invasive phenotype. BMC Cancer 14: 276, 2014.

25. Ma X, Gao Y, Fan Y, Ni D, Zhang Y, Chen W, Zhang P, Song E, Huang Q, Ai Q, et al: Overexpression of E2F1 promotes tumor malignancy and correlates with TNM stages in clear cell renal cell carcinoma. PLoS One 8: e73436, 2013.

26. Engelmann D, Mayoli-Nüssle D, Mayrhofer C, Fürst K, Alla V, Stoll A, Spitschak A, Abshagen K, Vollmar B, Ran S, et al: E2F1 promotes angiogenesis through the VEGF-C/VEGFR-3 axis in a feedback loop for cooperative induction of PDGF-B. J Mol Cell Biol 5: 391-403, 2013.

27. Lee J, Park CK, Park JO, Lim T, Park YS, Lim HY, Lee I, Sohn TS, Noh JH, Heo JS, et al: Impact of E2F-1 expression on clinical outcome of gastric adenocarcinoma patients with adjuvant chemoradiation therapy. Clin Cancer Res 14: 82-88, 2008.

28. Grupp K, Höhne TS, Prien K, Hube-Magg C, Tsourlakis MC, Sirma H, Pham T, Heinzer H, Graefen M, Michl U, et al: Reduced CD147 expression is linked to ERG fusion-positive prostate cancers but lacks substantial impact on PSA recurrence in patients treated by radical prostatectomy. Exp Mol Pathol 95: 227-234, 2013.

29. Pértega-Gomes N, Vizcaíno JR, Miranda-Gonçalves V, Pinheiro C, Silva J, Pereira H, Monteiro P, Henrique RM, Reis RM, Lopes C, et al: Monocarboxylate transporter 4 (MCT4) and CD147 overexpression is associated with poor prognosis in prostate cancer. BMC Cancer 11: 312, 2011.

30. Zhong WD, Han ZD, He HC, Bi XC, Dai QS, Zhu G, Ye YK, Liang YX, Qin WJ, Zhang Z, et al: CD147, MMP-1, MMP-2 and MMP-9 protein expression as significant prognostic factors in human prostate cancer. Oncology 75: 230-236, 2008.

31. Han ZD, Bi XC, Qin WJ, He HC, Dai QS, Zou J, Ye YK, Liang YX, Zeng GH, Chen ZN, et al: CD147 expression indicates unfavourable prognosis in prostate cancer. Pathol Oncol Res 15: 369-374, 2009. 